\title{
Running the hypothesis of a bio molecular approach to psychiatric disorder characterization and fatty acids therapeutical choices
}

\author{
Massimo Cocchi ${ }^{1,2^{*}}$, Lucio Tonello ${ }^{2}$ \\ From $1^{\text {st }}$ International Congress on Neurobiology and Clinical Psychopharmacology and European \\ Psychiatric Association Conference on Treatment Guidance \\ Thessaloniki, Greece. 19-22 November 2009
}

Beyond the conviction that Major Depression can found its origin in genetics [1-3] a bio molecular mechanism could be hypothesized from what emerged from the studies on platelets fatty acid composition in human (normal and depressive subjects) which allowed classifying the depressive disorder [4] using an Artificial Neural Network (Self Organizing Map-SOM) [5], as mathematical tool, because of the complexity of the membrane dynamics.

Rapid changes in membrane lipid composition or in the cytoskeleton could modify neuronal signalling. In the knowledge to have found something that could have implications in the understanding of some aspects of psychiatric disorders and a very suggestive hypothesis was build as summarized in Figure 1. In figure 1 is described the molecular depression hypothesis made according to Cocchi and collegues [4], Donati and collegues [6], Hameroff and Penrose [7]. The membrane viscosity can modify the Gs $\alpha$ protein status. The Gs $\alpha$ protein is connected with Tubulin. Tubulin, depending on local membrane lipid fase concentration, may serve as a positive or negative regulator of phosphatidylinositol bisphosphate (PIP2) hydrolysis, such as Gs $\alpha$ protein does. Tubulin is known to form high-affinity complexes with certain $G$ proteins. The formation of such complexes allows tubulin to activate Gs $\alpha$, which, in turn, can activate the Protein Kinase $\mathrm{C}$ and fosters a system whereby elements of the cytoskeleton can influence Gprotein signalling. Rapid changes in membrane lipid composition or in the cytoskeleton might modify neuronal signalling. We have hypothesized that through this mechanism is possible to modify the consciousness state and that it is mesurable through gamma syncrony EEG.

There are strong reasons to think that each fatty acid combination of Palmitic Acid (PA), Linoleic Acid (LA) and Arachidonic Acid (AA), in platelet, is responsible of the membrane viscosity and, therefore, of the molecular conditioning of the cellular stuctures (Gs $\alpha$ and Tubulin) and that the main therapeutic target is the reduction of the Arachidonic Acid.

\section{Author details \\ ${ }^{1}$ DIMORFIPA, University of Bologna, Italy. ${ }^{2}$ Faculty of Human and \\ Technological Sciences, LUdES University, Lugano, Switzerland.}

Published: 22 April 2010

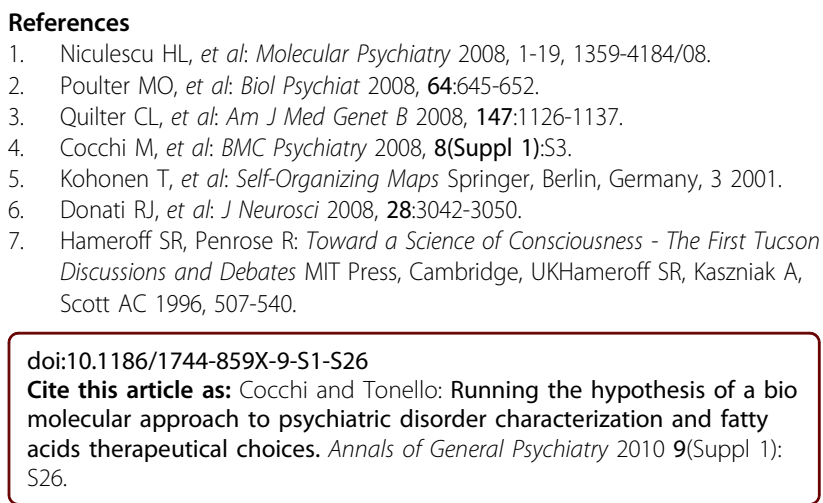


Massimo Cocchi

Lucio Tonello

Mark Rasenick

Stuart Hameroff

LIFE

Counsciousness
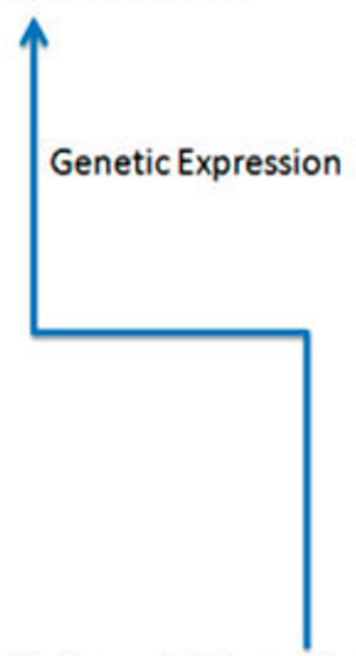

Mathematic Model Not

Linear
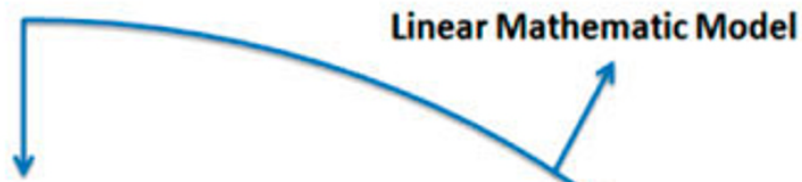

$<$

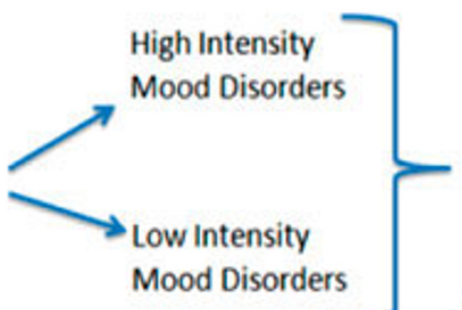

Tubulin

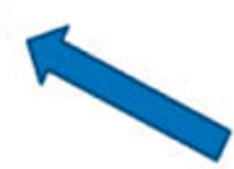

Raft Lipid Microdomain

GS $\alpha$ Protein
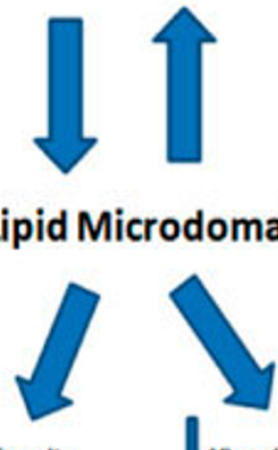

Viscosity

Viscosity

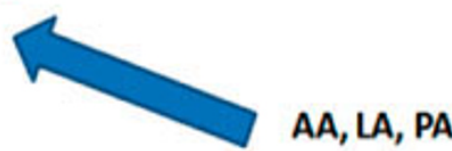

Membrane Fatty Acids 